\title{
Guided Wave Propagation Study on Laminated Composites by Frequency-Wavenumber Technique
}

\author{
Zhenhua Tian ${ }^{1}$, Lingyu Yu ${ }^{1 *}$, Cara A.C. Leckey ${ }^{2}$ \\ ${ }^{1}$ Mechanical Engineering Department, University of South Carolina, Columbia, SC \\ ${ }^{2}$ Nondestructive Evaluation Sciences Branch, NASA Langley Research Center, Hampton, VA
}

\begin{abstract}
Toward the goal of delamination detection and quantification in laminated composites, this paper examines guided wave propagation and wave interaction with delamination damage in laminated carbon fiber reinforced polymer (CFRP) composites using frequency-wavenumber $(f-k)$ analysis. Three-dimensional elastodynamic finite integration technique (EFIT) is used to acquire simulated time-space wavefields for a CFRP composite. The time-space wavefields show trapped waves in the delamination region. To unveil the wave propagation physics, the time-space wavefields are further analyzed by using two-dimensional (2D) Fourier transforms (FT). In the analysis results, new $f$ - $k$ components are observed when the incident guided waves interact with the delamination damage. These new $f-k$ components in the simulations are experimentally verified through data obtained from scanning laser Doppler vibrometer (SLDV) tests. By filtering the new $f-k$ components, delamination damage is detected and quantified.
\end{abstract}

Keywords: Guided waves, frequency wavenumber analysis, EFIT simulation, delamination quantification

\section{INTRODUCTION}

Current composite "safety margin" design comes with a weight penalty which could be mitigated by the development of accurate, efficient, and reliable structural health monitoring (SHM) methods to locate and quantify the unique damage types that can occur in composite materials. Unexpected damage can occur in aerospace composites due to impact events or due to stressing of the material during off-nominal loading events. Matrix cracking, fiber breakage, fiber-matrix debonding, and delamination are the most common types of damage in fiber composites [1]. In particular, laminated composites are especially susceptible to delamination damage due to their weak transverse tensile and interlaminar shear strengths (as compared to their in-plane properties)[1]. Delamination damage can occur internally such that it is not readily visible on the composite surface [2]. Hidden delamination damage caused by the impacts must be detected and evaluated before it becomes critical. The problem of how best to detect such material changes in laminated composite components is still being solved, and a solution to this problem will be imperative for safe and functional, optimally-designed, next-generation composite aircraft.

Guided ultrasonic waves have proved effective for SHM due to their ability to inspect large areas while maintaining sensitivity to small defects in the structure; and also due to the capability to implement guided wave techniques using permanently installed, lightweight low-profile piezoelectric wafer sensors [3-9]. Advances in guided wave based SHM technologies over the last decade have demonstrated the feasibility of detecting and locating damage in composite structural components [10-19]. Due to the complex wave interaction that occurs when hidden delamination damage is present, tremendous efforts have been put into delamination detection methodologies. Guo and Cawley studied the interaction of the $\mathrm{S}_{0}$ Lamb mode with delaminations [20], showing that the reflection amplitude of the $\mathrm{S}_{0}$ mode from a delamination is strongly dependent on the delamination depth. Petculescu et al. used a group delay measurement technique for the detection and sizing of delaminations in unidirectional and cross-ply composites [21]. They found that the delamination affects the traveling time of ultrasonic wave packet. Staszewski et al. used 3-D laser vibrometry to locate delamination damage and estimate its severity in a composite plate, demonstrating that the delamination can be revealed by the amplitude profiles of Lamb waves [2]. Purekar and Pines demonstrated the application of piezoelectric phased sensor arrays to detect the presence of delamination damage in a composite laminate [22]. Michaels et al. used guided wavefield images and frequency-wavenumber domain analysis to study the wave

\footnotetext{
*Corresponding author. Email: yu3@cec.sc.edu
} 
interactions with structural discontinuities in composites [23]. Yeum et al. presented a delamination detection technique using a dual piezoelectric transducer network [24]. The result for the case they studied showed that the anti-symmetric $\mathrm{A}_{0}$ mode slowed down when it passed through a delamination region, while the speed of the symmetric $\mathrm{S}_{0}$ mode was little affected by the delamination.

All of this prior work demonstrated that delamination damage affects various characteristics of the interrogation waves (such as amplitude and travelling time). In addition, the damage can cause wave scattering, mode conversion, and multiple reflections in the delamination region. To understand the mechanisms of wave propagation and interactions in the delamination region, other researchers studied the mechanisms through simulation and experiment. Hayashi and Kawashima reported the use of numerical simulations to study guided wave propagations and interactions in delaminated regions [25]. Multiple reflections in the delamination area were confirmed. Hence, a considerable amount of ultrasonic energy was "trapped" above and below delaminated regions until eventually being dissipated. Ramadas et al. studied the interaction of the anti-symmetric $\mathrm{A}_{0}$ mode with symmetric delaminations [26]. They also identified multiple reflections in the delamination region. Sohn et al. have studied the trapped phenomenon of guided wave propagation in delaminated composites through the use of wavefield images obtained from a SLDV[27, 28]. It was observed that upon entering the delaminated region, the incident waves would split above and below the delamination and then propagate independently through upper and lower lamina (single delamination scenario being assumed). After that a significant portion of these waves were reflected back from the far edge (exit region). The reflected waves then pass back above (or below) the delaminated area and experience reflections at the original entrance. The process was repeated until the energy was dissipated, as found in the simulation work reported by Hayashi and Kawashima [25]. Glushkov et al. also observed similar wave energy trapping and localization in a strip delamination by using laser vibrometry to record the wavefield [29]. Despite these advances, guided wave techniques for composites are still insufficient in regards to a quantitative understanding of the mechanisms of wave interactions with delaminations in composites.

In this paper, we examine guided wave propagation and interaction in the delamination region in the carbon fiber reinforced polymer (CFRP) composites by using $f-k$ analysis for the purpose of delamination detection and quantification. Elastodynamic finite integration technique (EFIT) simulations and SLDV experiment are both used to obtain the time-space wavefields regarding the wave interactions with the delamination damage. The $f-k$ analysis is implemented to expand the level of details such as $f-k$ components and wave mode contents of the wavefields. The $f-k$ filtering technique is used to detect and quantify the delamination. This paper is organized as follows. Section 2 presents the guided wave simulation technique that was implemented to acquire in-plane and out-of-plane wavefields, and compares the wavefields between the pristine and delaminated plates. Section 3 describes the $f-k$ analysis technique which was used to analyze the wavefields, and compares the analysis results between the pristine and delaminated plates. Section 4 presents experimental tests to verify the wavefields and $f-k$ analysis results of the EFIT data. Section 5 presents the $f-k$ filtering which is used for delamination detection and quantification. A summary of findings is provided in Section 6.

\section{EFIT WAVE MOTION SIMULATION}

Computational wave simulation has proven to be an effective tool for clarifying complex ultrasonic wave phenomenon without labor-consuming and costly experimental exploration. Previous work by the authors has demonstrated how 3D simulated guided wave data can aid in the study of guided wave interaction with damage in isotropic and anisotropic materials [19, 30, 31]. In this work, 3D EFIT was implemented to acquire guided wave propagation data for laminated composites, including cases of wave interaction with the delamination damage. For composite materials, EFIT can incorporate ply-level material property changes; thus allowing for specific CFRP layups to be accurately simulated. The code is custom parallelized to run efficiently on cluster and multicore computing resources. Additional details about the anisotropic EFIT simulation code, including results of code validation studies for pristine composite plates, can be found in prior work [19]. The complete EFIT equations can be found in reference [32]. Note that for the EFIT model implemented for this paper, 3D refers to the three Cartesian spatial dimensions, and that the wave propagation data generated by the simulation are 4D including time. 


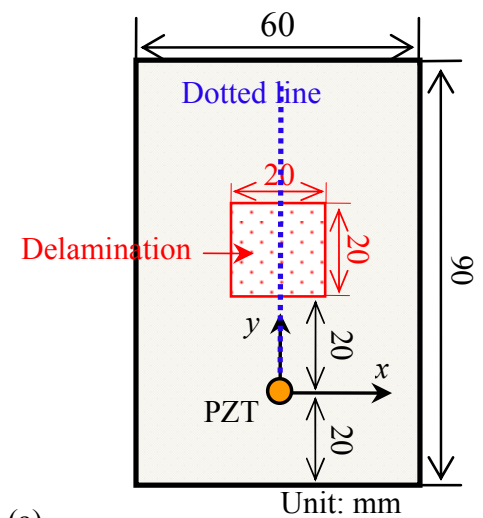

(a)

Figure 1

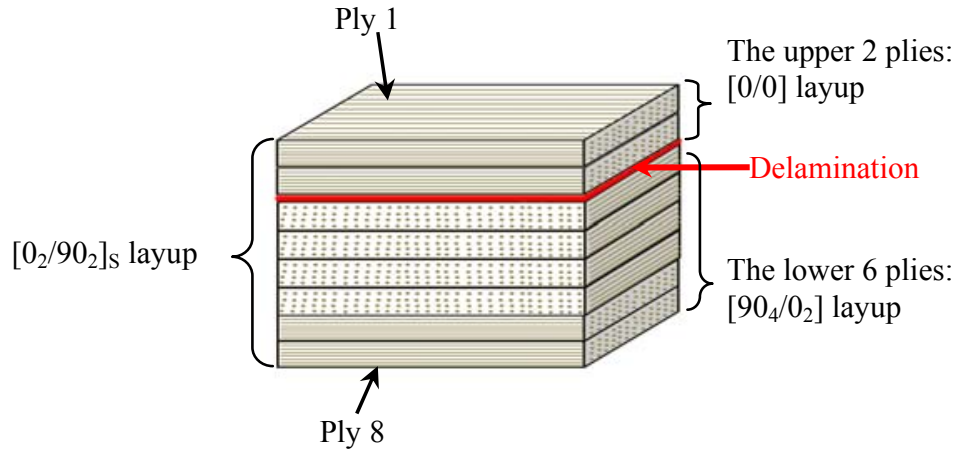

(b)

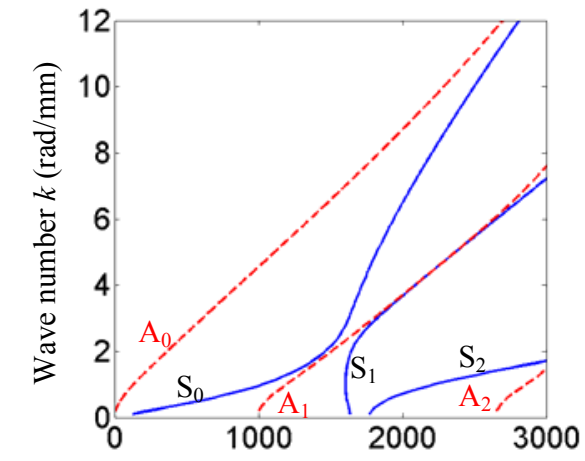

(a)

EFIT simulation configuration: (a) the simulation layout; (b) the side view of the $\left[0_{2} / 90_{2}\right]_{\mathrm{S}}$ layup.

Figure 2. Dispersion curves of the 8-ply IM7/8552 composite laminate with $\left[0_{2} / 90_{2}\right]_{\mathrm{s}}$ layup: (a) $f$ - $k$ curves for $90^{\circ}$ direction; (b) group velocity curves for $90^{\circ}$ direction.

Table 1 Material properties

\begin{tabular}{lllllllllll}
\hline$\rho\left(\mathrm{kg} / \mathrm{m}^{3}\right)$ & $\mathrm{E}_{1}(\mathrm{GPa})$ & $\mathrm{E}_{2}(\mathrm{GPa})$ & $\mathrm{E}_{3}(\mathrm{GPa})$ & $\mathrm{G}_{12}(\mathrm{GPa})$ & $\mathrm{G}_{13}(\mathrm{GPa})$ & $\mathrm{G}_{23}(\mathrm{GPa})$ & $v_{12}$ & $v_{13}$ & $v_{23}$ & Thickness $(\mathrm{mm})$ \\
\hline 1570 & 171.4 & 9.08 & 9.08 & 5.29 & 5.29 & 2.80 & 0.32 & 0.32 & 0.5 & 0.1068 \\
\hline
\end{tabular}

\subsection{EFIT simulation setup}

In this work wave propagation in an 8-ply IM7/8552 composite laminate plate with layup $\left[0_{2} / 90_{2}\right]_{\mathrm{s}}$ (Figure 1b) is studied. The total thickness of the simulated laminate is $0.854 \mathrm{~mm}$. The material properties for a single ply are given in Table 1, where $E_{1}$ is in the fiber direction (and where $v$ is Poisson's ratio). With the composite layup and material properties, the dispersion curves along $90^{\circ}$ (corresponding to the direction along the dotted line in Figure 1a) were calculated using DISPERSE software [33]. The calculated dispersion curves are plotted in Figure 2. The dispersion curves show that below the cutoff frequency $1 \mathrm{MHz}$ there are two guided wave modes existing in the plate, the fundamental $\mathrm{A}_{0}$ mode (with lower velocity) and $\mathrm{S}_{0}$ mode (with higher velocity).

Both the pristine and damaged plates were modeled by using the EFIT. To make a simple case for preliminary study, the damage is a $20 \mathrm{~mm} \times 20 \mathrm{~mm}$ delamination between the uppermost $0 / 90$ plies as illustrated in Figure $1 \mathrm{~b}$. Prior work by Leckey et al. has demonstrated that delamination geometries can be quite complex (depending on the composite layup and damage severity), and the simple damage case studied in this work is considered to be a starting point for developing a quantitative understanding of in-plane and out-of-plane guided wave interaction with delaminations in composites [19,34].

In order to accurately capture the small wavelengths of wave modes that may exist in the layers (above or below the delamination as illustrated in Figure $1 b$ ) which will have much smaller thickness, a small spatial step size was enforced for the EFIT simulation. The spatial step size was set to $\Delta x=20 \mu \mathrm{m}$ for both the pristine and delaminated cases. 
It is important to note that EFIT requires a spatial step size no larger than $\Delta \mathrm{x} \leq \lambda_{\min } / 10$, and for cases where delaminations (or a change in thicknesses) are present, a $\lambda_{\min }$ based on the full plate thickness may not suffice. The delamination was incorporated by implementing stress free boundary conditions at the delamination location. Excitation used in the model is a $300 \mathrm{kHz} 3$-count Hanning window smoothed sine wave. The excitation was introduced to the simulation space as a 7 $\mathrm{mm}$ diameter ring-type excitation on the top laminate surface in order to approximate the behavior of a $7 \mathrm{~mm}$ bonded piezoelectric wafer [30]. Velocity data as a function of time $(t)$ and space $(x, y)$ from the top surface of the composite plates, including in-plane $v_{x}$ and $v_{y}$ components as well as out-of-plane $v_{z}$ component, are generated from the EFIT simulation.
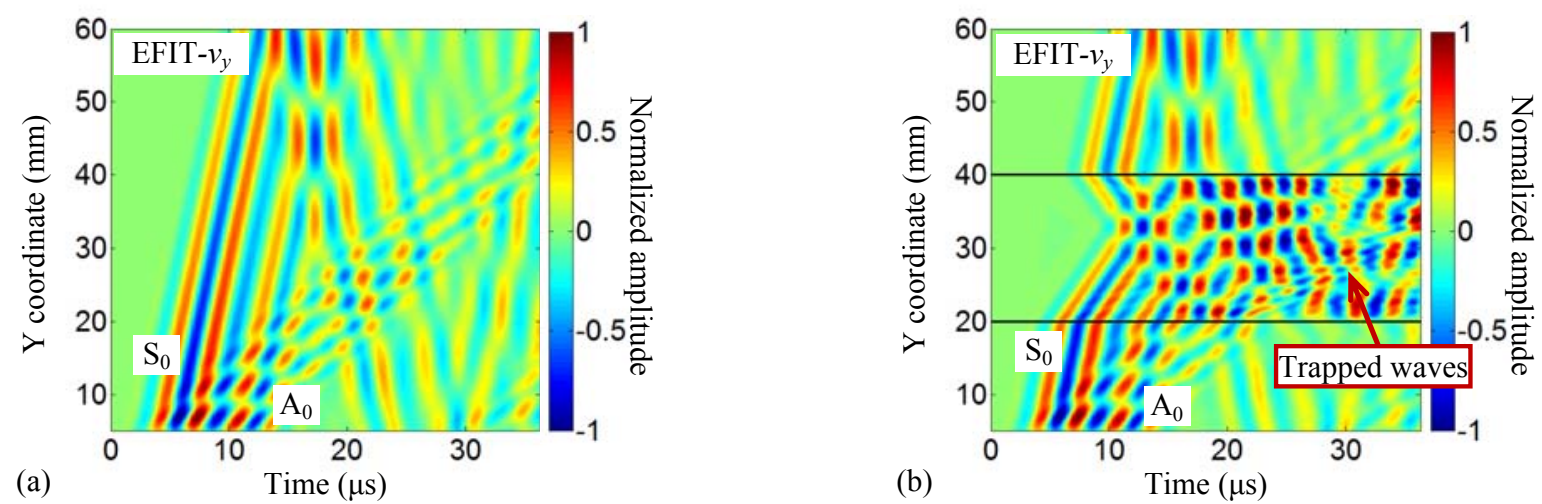

Figure 3. The time-space wavefields of EFIT in-plane $v_{y}$ components: (a) for the pristine plate; (b) for the plate with a delamination.
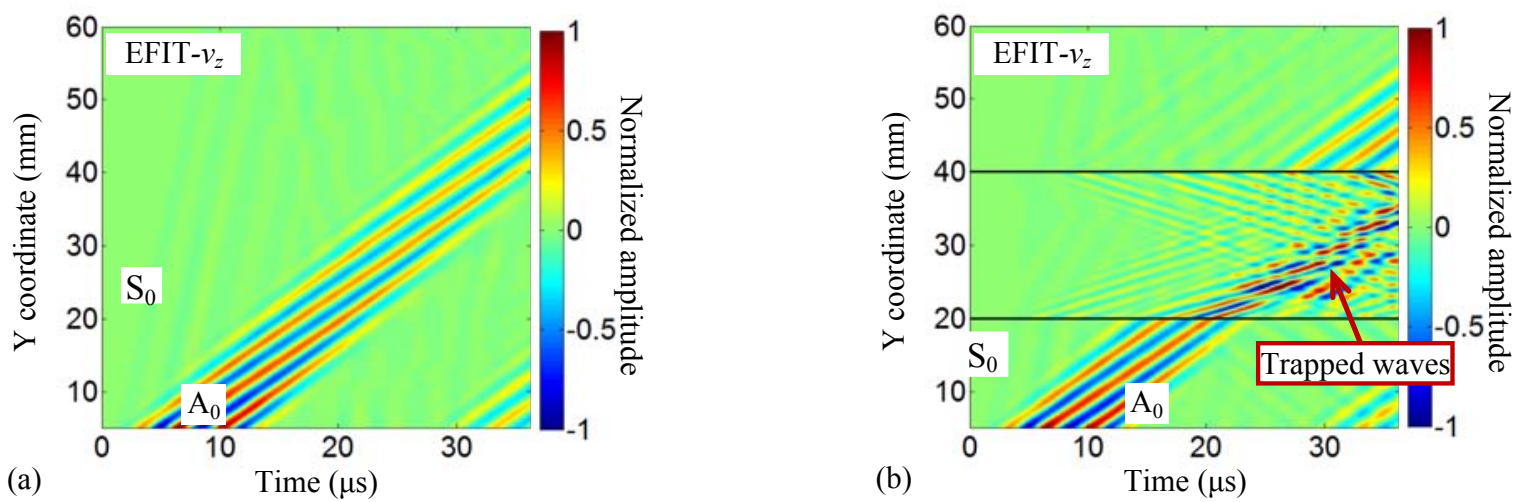

Figure 4. The time-space wavefields of EFIT out-of-plane $v_{z}$ components: (a) for the pristine plate; (b) for the plate with a delamination. The out-of-plane component of $\mathrm{A}_{0}$ is prevailing compared to that of $\mathrm{S}_{0}$.

\subsection{Simulation results}

To study the wave interaction with the delamination, the EFIT simulation results along the $y$ axis from the source through the delaminated region (dotted line in Figure 1a) are used. The selected simulation results are two dimensional (2D) in terms of argument $y$ (propagation distance) and argument $t$ (time), denoted as time-space wavefield $v(t, y)$. The $v(t, y)$ notation refers to velocity wavefield as either in-plane, $v_{x}(t, y)$ and $v_{y}(t, y)$, or out-of-plane, $v_{z}(t, y)$, components. The in-plane $v_{x}$ component is zero in this study due to the fact that the $v_{x}$ component is normal to the wave propagation direction along the $y$ axis $\left(90^{\circ}\right.$ direction). Hence, only the in-plane $v_{y}$ and out-of-plane $v_{z}$ components will be analyzed and discussed in this paper.

Figure $3 \mathrm{a}$ and $\mathrm{b}$ show the time-space wavefields of the in-plane $v_{y}$ components for the pristine and delaminated plates, respectively. A faster $\mathrm{S}_{0}$ mode and a slower $\mathrm{A}_{0}$ mode, as well as their interactions with the structural discontinuities (plate boundary and delamination for the delaminated cases), can be clearly viewed. The in-plane $\mathrm{S}_{0}$ mode shows higher amplitude than that of the $\mathrm{A}_{0}$ mode. It is seen from the wavefields that in the delamination region, 
waves bounce back and forth between the boundaries of the delamination, distinctively exhibiting the "trapped waves" phenomenon. The observed behavior agrees with the wavefield observations presented by Sohn et al [27].

Figure $4 \mathrm{a}$ and $\mathrm{b}$ show the time-space wavefields of the out-of-plane $v_{z}$, components for the pristine and delaminated plates, respectively. It is seen the out-of-plane $\mathrm{A}_{0}$ mode has higher amplitude than the $\mathrm{S}_{0}$ mode. The "trapped waves" phenomenon is also observed in the out-of-plane wave interactions with the boundaries of the delamination, similar to that of the in-plane $v_{y}$ observations.

\section{TRAPPED WAVE MODES WITHIN DELAMINATIONS}

In the above section, the time-space wavefield shows wave interactions with the delamination and exhibits the trapped waves in the delamination region. However, implicit wave propagation characteristics such as $f-k$ components and wave mode contents of the time-space wavefield are not readily seen. It has been demonstrated that wavefield analysis representing the $f-k$ content has abundant information regarding the existence of various wave modes and wave propagation characteristics [23, 35-37]. Thus, in this section we transform the time-space wavefield to the $f-k$ representation to analyze the wave interactions with the delamination.

\section{1 $\quad F-k$ analysis}

The $f-k$ representation can be obtained from the 2D time-space wavefield data by applying the 2D FT, mathematically given as $[23,35-38]$

$$
U(f, k)=\mathrm{F}_{2 \mathrm{D}}[u(t, x)]=\int_{-\infty}^{\infty} \int_{-\infty}^{\infty} u(t, x) e^{-j(2 \pi f t-k x)} d t d x
$$

where $U(f, k)$ is the resulting $f-k$ representation or "spectrum" in terms of the frequency variable $f$ and the wavenumber variable $k$. The $U(f, k)$ can be interpreted as an alternative representation of the time-space wavefield $u(t, x)$. The $f-k$ spectrum not only shows the $f-k$ components of the wavefield but also provides an easy means to identify the wave mode compositions.

\subsection{In-plane component $\mathrm{v}_{\mathrm{y}}$ analysis}

The $f-k$ spectrum of the EFIT in-plane $v_{y}$ wavefield for the pristine plate is first calculated and plotted in Figure 55a. Theoretical dispersion curves for waves in the $90^{\circ}$ direction in the $\left[0_{2} / 90_{2}\right]_{\mathrm{s}}$ laminate are plotted in the same figure as dotted $\left(\mathrm{A}_{0}\right)$ and solid $\left(\mathrm{S}_{0}\right)$ lines. The pristine plate $f$ - $k$ spectrum shows the lower amplitude $\mathrm{A}_{0}$ and higher amplitude $\mathrm{S}_{0}$ modes which match well with the theoretical dispersion curves.

In the delaminated plate, the delamination damage, which splits the originally bonded full laminate into two parts (above and below the delamination as illustrated in Figure 1b), will result in waves propagating in each section. Since the two parts, above and below the delamination, will have different layups and thicknesses compared to the original pristine plate, waves measured at an outer plate surface region above (or below) the delamination will have modified propagation characteristics and wavenumbers. As expected, the delaminated plate spectrum (Figure $5 b$ ) shows new $f-k$ components in between the original $\mathrm{A}_{0}$ and $\mathrm{S}_{0}$ modes, including both positive (forward propagating) and negative (backward propagating) counterparts. We compared the new $f-k$ components with the dispersion curves for the material above the delamination, equivalent to a two ply [0\0] laminate (the corresponding dispersion curves are plotted in 
Figure6), since only the data of the top plate surface was used for analysis. The comparison in Figure $5 \mathrm{~b}$ shows, the new $f-k$ components match well with the theoretical curves of $\mathrm{S}_{0}$ mode in the two ply [0\0] laminate.
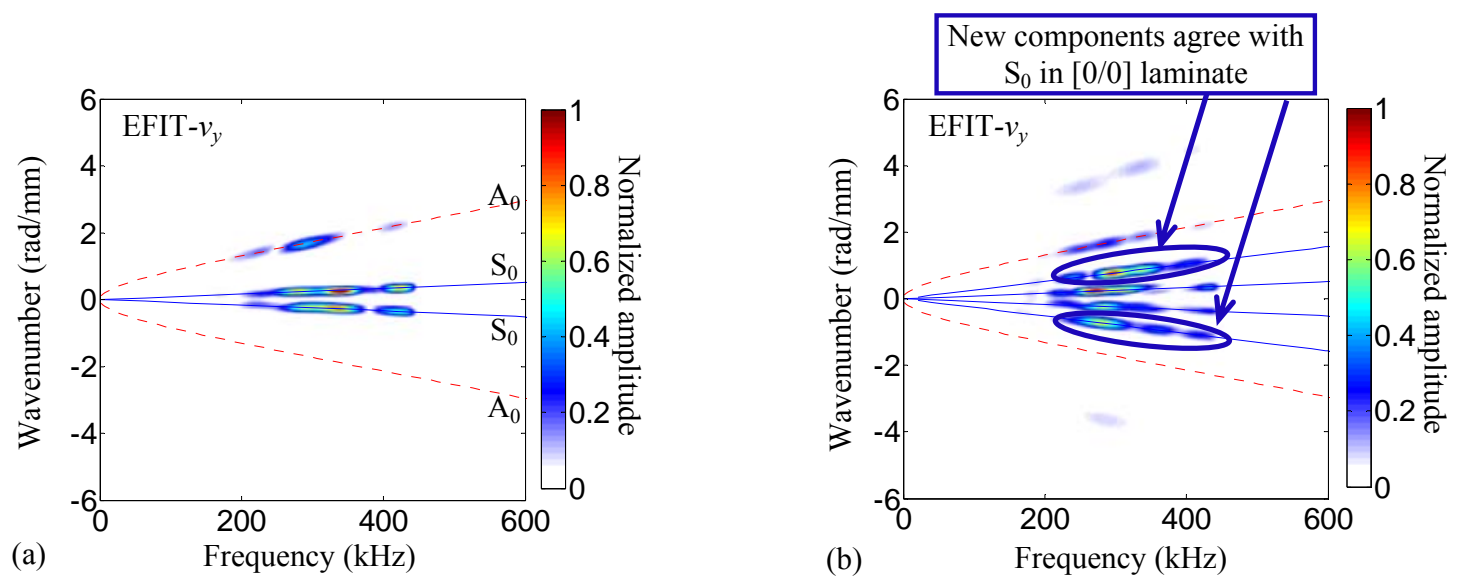

Figure 5. The $f-k$ spectra of EFIT in-plane $v_{y}$ components: (a) for the pristine plate; (b) for the plate with a delamination. Solid and dotted lines are theoretical dispersion curves for $\mathrm{S}_{0}$ and $\mathrm{A}_{0}$ wave modes, respectively.
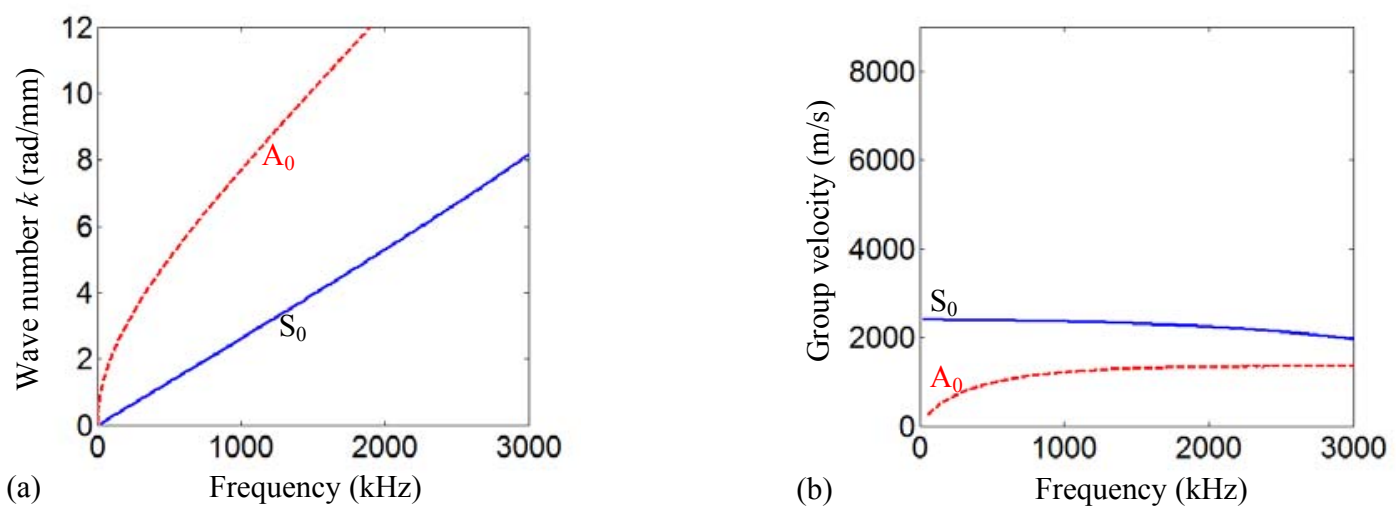

Figure 6. Dispersion curves for a 2 ply laminate with [0/0] layup: (a) $f-k$ curves for $90^{\circ}$ direction; (b) group velocity curves for $90^{\circ}$ direction.
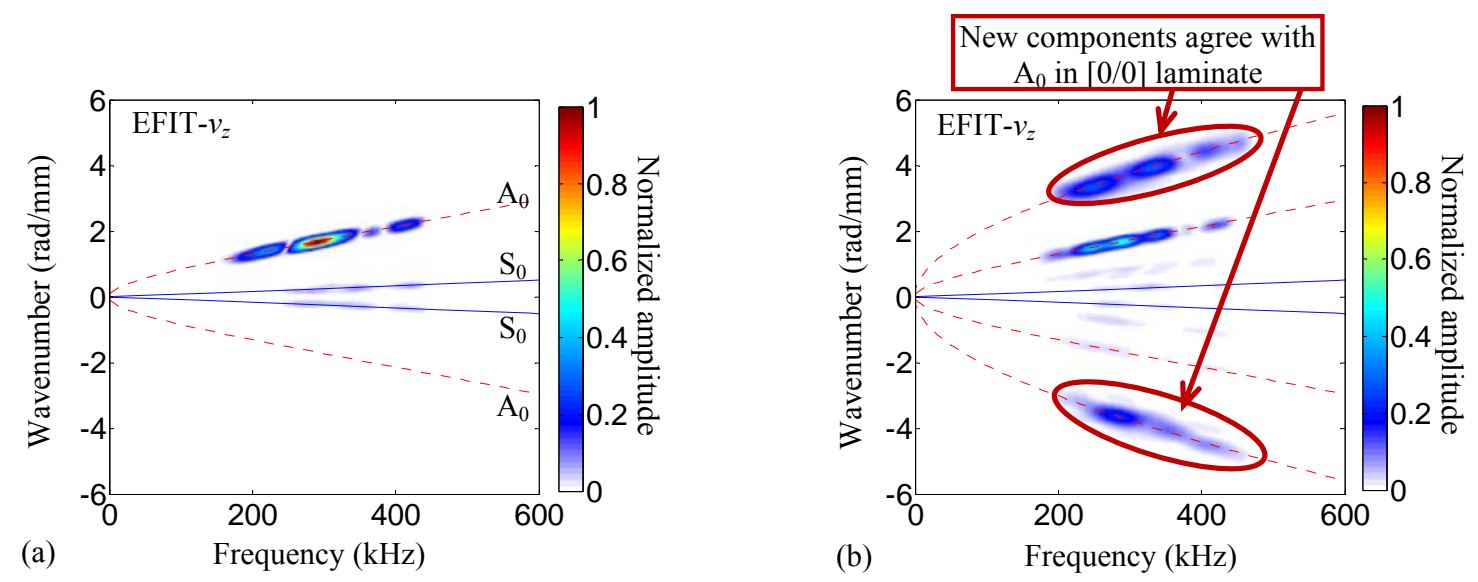
Figure 5. The $f-k$ spectra of EFIT out-of-plane $v_{z}$ components: (a) for the pristine plate; (b) for the plate with a delamination. Solid and dotted lines are theoretical dispersion curves for $\mathrm{S}_{0}$ and $\mathrm{A}_{0}$ wave modes, respectively.

\subsection{Out-of-plane component $\mathrm{v}_{\mathrm{z}}$ analysis}

Figure 5a plots the pristine plate $f-k$ spectrum for the EFIT out-of-plane $v_{z}$ wavefield. The pristine spectrum shows higher amplitude $\mathrm{A}_{0}$ and lower amplitude $\mathrm{S}_{0}$ modes which match well with the theoretical dispersion curves. When the delamination is introduced, however, new $f-k$ components (positive and negative) appear in the delaminated plate spectrum (Figure $5 \mathrm{~b}$ ), and match well with the theoretical curves of $\mathrm{A}_{0}$ mode in the two ply [0\0] laminate. The analysis results of the out-of-plane $v_{z}$ wavefields further confirm that the new $f-k$ components appear in the spectrum of the delaminated plate, and are correlated to the waves propagating in the delamination region.

\section{EXPERIMENTAL VERIFICATION}

In sections 2 and 3, the EFIT data show interesting results such as delamination induced wave interactions, "trapped waves" and new $f-k$ components. To verify these observations from the EFIT data, this section presents comparisons between the out-of-plane EFIT data and experimental data acquired using a 1D scanning laser Doppler vibrometer (SLDV).

\subsection{Experimental setup}

Two $380 \mathrm{~mm} \times 380 \mathrm{~mm}$ 8-ply IM7/8552 composite laminate plates with layup $\left[0_{2} / 90_{2}\right]_{\mathrm{s}}$ (the same layup and material as used in the EFIT model) were fabricated at NASA Langley. One is a pristine plate and the other is a delaminated plate. The delamination is generated by inserting a $20 \mathrm{~mm} \times 20 \mathrm{~mm}$ Teflon film under the second ply during the ply lay-up process. Figure 6 shows the experimental setup. Guided waves in the plates are excited by a surface bonded PZT actuator (APC 851: $7 \mathrm{~mm}$ diameter, $0.2 \mathrm{~mm}$ thickness) located at the coordinate $(0,0)$. The excitation signal, a $300 \mathrm{kHz} 3$-cycle Hanning window smoothed sine wave, is generated by an arbitrary waveform function generator (Hewlett Packard 33120A). The signal is amplified to $40 \mathrm{~V}$ by a voltage amplifier (Krohn-Hite 1506). A SLDV (Model: Polytec PSV-400-M2) is used to acquire high spatial resolution velocity wavefield data experimentally. This Polytec model is a 1D SLDV, and therefore only the motion along the laser beam can be measured. In the test, the laser beam is set normal to the surface of the specimen such that the out-of-plane velocity is measured (experimental $v_{z}$ ). Scanning is performed along the dotted line (shown in Figure 6) from $5 \mathrm{~mm}$ to $60 \mathrm{~mm}$ away from the PZT actuator with a spatial resolution of $0.2 \mathrm{~mm}$. The SLDV data are used to validate the simulation results in terms of the out-of-plane velocity $v_{z}$.

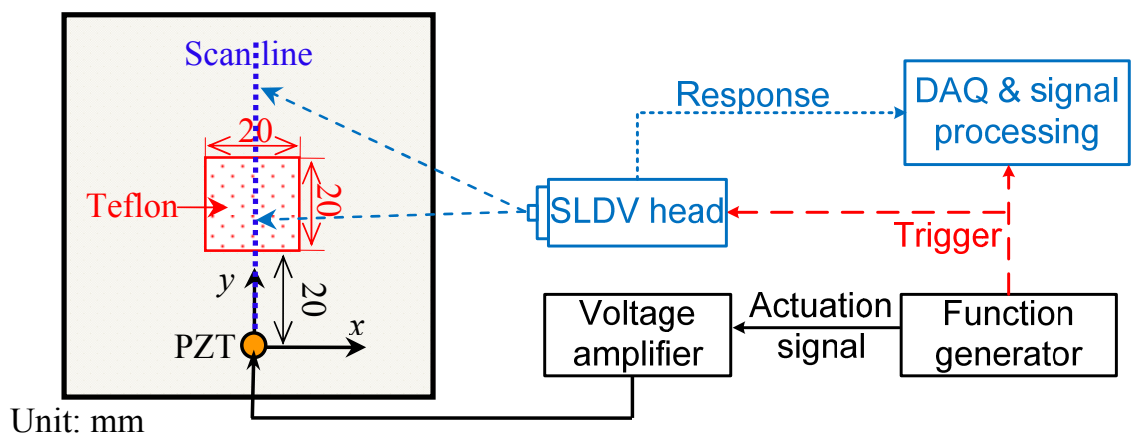

Figure 6. The schematic of laser vibrometry experimental test setup.

\subsection{Experimental results}

Figure $7 \mathrm{a}$ and $\mathrm{b}$ show the time-space wavefields of SLDV measurements $\left(v_{z}\right)$ for the pristine and delaminated plates, respectively. It is seen the $\mathrm{A}_{0}$ mode has higher amplitude than the $\mathrm{S}_{0}$ mode. The experimental wavefield of the delaminated plate shows waves propagating back and forth (as standing waves) between the boundaries of the delamination and exhibit the "trapped waves" phenomenon. The experimental wavefields (Figure 7) verify the observations from EFIT out-of-plane wavefields ( 
Figure 4), such as delamination induced wave interactions and "trapped waves" phenomenon.

Figure $8 \mathrm{a}$ and $\mathrm{b}$ show the $f-k$ spectra of the SLDV measurements for the pristine and delaminated plates, respectively. Again, the pristine spectrum shows higher amplitude $\mathrm{A}_{0}$ and lower amplitude $\mathrm{S}_{0}$ modes. Note that the EFIT results in figures 4 and 7 showed even lower amplitude for the $S_{0}$ mode. It is expected that this difference between EFIT and SLDV data is due to the idealized excitation used in EFIT compared to the real excitation in the SLDV case. When the delamination is introduced, new $f-k$ components (positive and negative) appear in the delaminated plate spectrum (Figure $8 \mathrm{~b}$ ), and match well with the theoretical curves of $\mathrm{A}_{0}$ mode in the two ply [0\0] laminate. The analysis results of the SLDV measurements are in overall agreement with the results of the EFIT data. The experimental $f-k$ spectra verify the new $f-k$ components observed in EFIT $f-k$ spectrum for the delaminated plate.
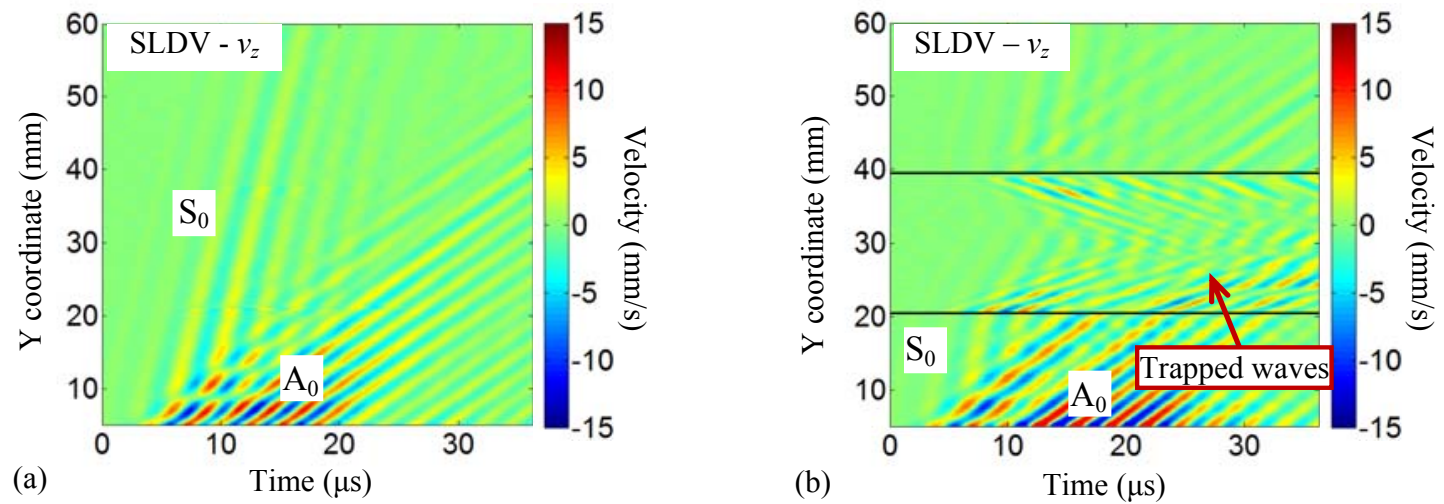

Figure 7. The time-space wavefields measured by the SLDV: (a) for the pristine plate; (b) for the plate with a delamination.
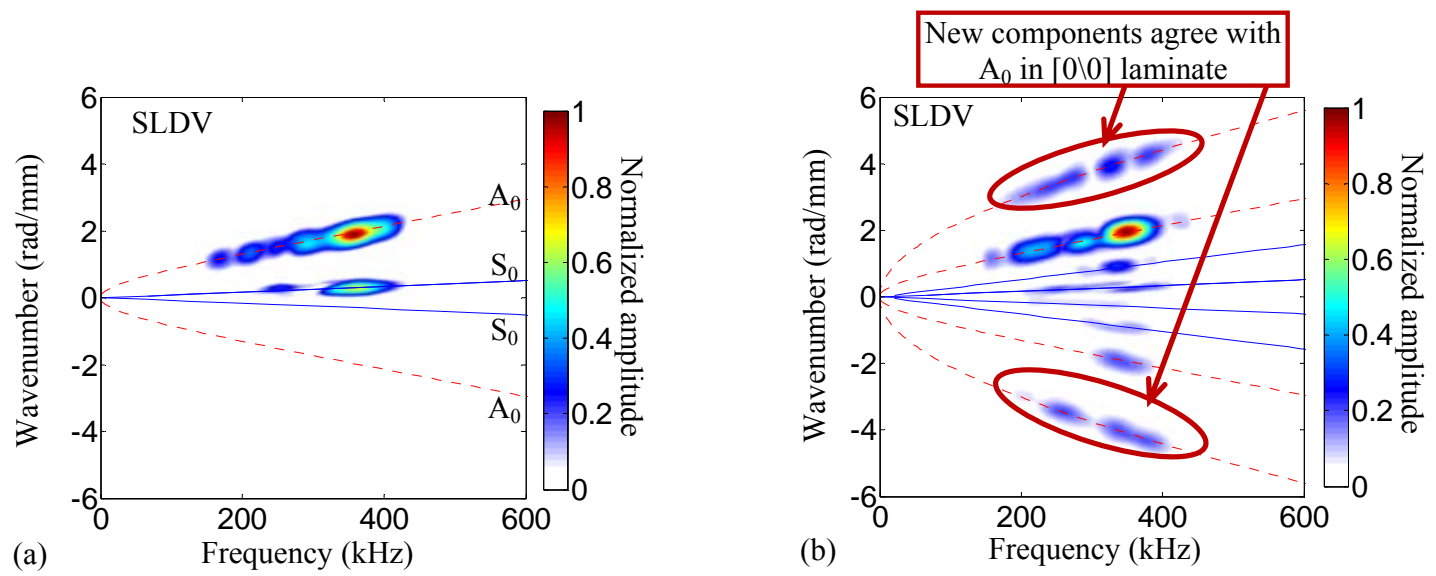

Figure 8. The $f-k$ spectra of SLDV measurements: (a) for the pristine plate; (b) for the plate with a delamination. Solid and dotted lines are theoretical dispersion curves for $\mathrm{S}_{0}$ and $\mathrm{A}_{0}$ wave modes, respectively. 


\section{DELAMINATION DETECTION AND QUANTIFICATION}

The $f-k$ analysis results of both EFIT simulations and SLDV measurements show new $f-k$ components appear in the spectra of the delaminated plate, and are correlated to the waves propagating in the delamination region. This section studies delamination detection and quantification by filtering the new $f-k$ components.

\section{$5.1 \quad f-k$ filtering}

The $f-k$ spectra show that various wave modes are discernible and separable in the $f-k$ domain. Thus, through filtering in the $f-k$ domain, any desired wave mode in the $f-k$ spectrum can be extracted $[23,36,39]$. The $f-k$ filtering process can be mathematically expressed as the product between the $f-k$ spectrum $U(f, k)$ and a $f-k$ filter function $W(f, k)$ as:

$$
U_{W}(f, k)=U(f, k) W(f, k)
$$

where $W(f, k)$ denotes the 2D filter in the $f-k$ domain and $U_{W}(f, k)$ is the filtered spectrum. The filtered spectrum $U_{W}(f, k)$ is then transformed back into the time-space domain by using the inverse 2D FT in order to obtain the filtered time-space wavefield $u_{W}(t, x)$ :

$$
u_{W}(t, x)=\mathrm{F}_{2 D}^{-1}\left[U_{W}\left(f, k_{x}\right)\right]
$$

To extract the waves in the delamination region, the $f-k$ filter $W(f, k)$ should be designed as a $2 \mathrm{D}$ bandpass filter which can keep the desired $f-k$ components correlated to the waves in the delamination region.

\subsection{Delamination detection by using in-plane $v_{y}$ component}

Figure 9a gives the spectral representation of the 2D bandpass $f-k$ filter with a Gaussian profile, which is intended to keep the new $f-k$ components, while removing all the rest in the EFIT $v_{y} f-k$ spectrum (Figure $5 b$ ). After $f-k$ filtering, the bandpass filtered spectrum with the new $f-k$ components is retained, as shown in Figure $9 \mathrm{~b}$. The filtered spectrum is then inversely transformed back into the time-space domain to reconstruct the wavefield. The reconstructed wavefield is presented in Figure 9c, showing that it represents waves propagating forward and backward between the delamination boundaries. The filtered wavefield, which only contains the waves in the delamination region, provides an easy means to detect and quantify the delamination damage. Details of $f-k$ filtering can be found in reference [39].
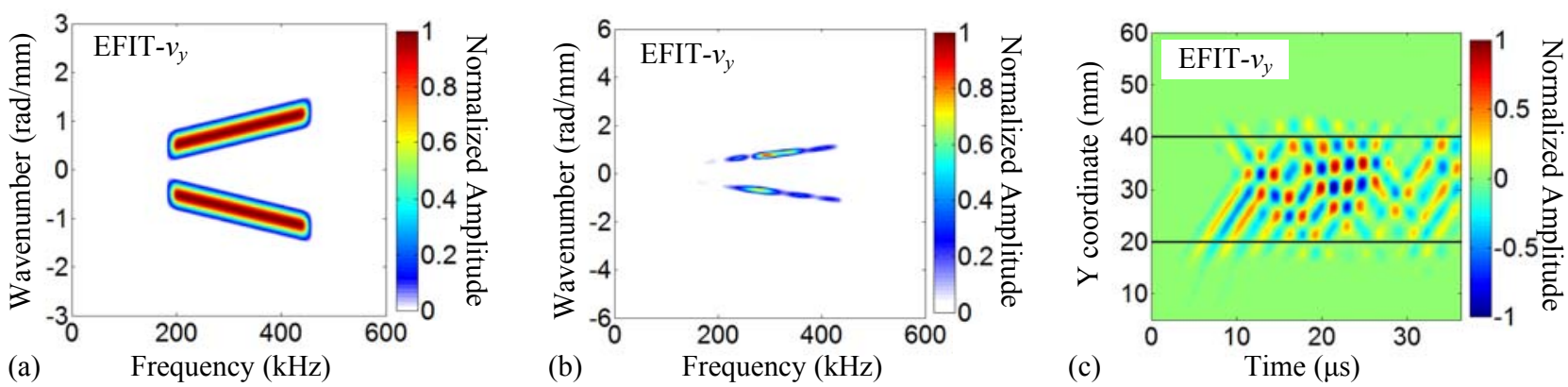

Figure 9. Delamination detection by using EFIT in-plane $v_{y}$ component: (a) the $f-k$ filter; (b) the filtered $f$ - $k$ spectrum;

(c) the filtered wavefield.

\subsection{Delamination detection by using out-of-plane $v_{z}$ component}

Since the out-of-plane data are also available from the SLDV tests, the $f-k$ filtering is applied to both EFIT and SLDV out-of-plane data. Figure $10 \mathrm{a}, \mathrm{b}$ and $\mathrm{c}$ show the $f-k$ filter, filtered spectrum and reconstructed time-space wavefield of the EFIT out-of-plane $v_{z}$ component. The reconstructed wavefield shows only the trapped waves propagating in the delamination region. Thus, from the reconstructed wavefield, we can detect and quantify the delamination location and size. In this case, from the wavefield in Figure $10 \mathrm{c}$, the delamination is detected at $y=30 \mathrm{~mm}$ with the width $20 \mathrm{~mm}$. The detection result is in consistent with the real delamination location.

Figure $11 \mathrm{a}, \mathrm{b}$ and $\mathrm{c}$ show the $f-k$ filter, filtered spectrum and reconstructed time-space wavefield of the SLDV data. The reconstructed wavefield shows only the trapped waves in the delamination region. From the reconstructed wavefield in Figure $11 \mathrm{c}$, the delamination is detected at $y=30 \mathrm{~mm}$ with the width $20 \mathrm{~mm}$. The detection result agrees well with the 
real delamination location and further confirms the feasibility of using $f-k$ filtering for delamination size quantification (for this analysis, which is 1D in space, the size is quantified in the y-direction length).

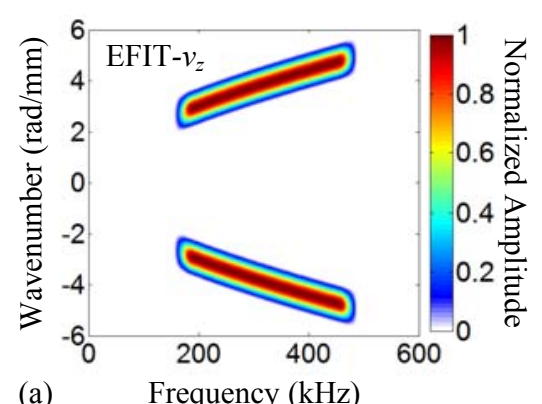

(a)

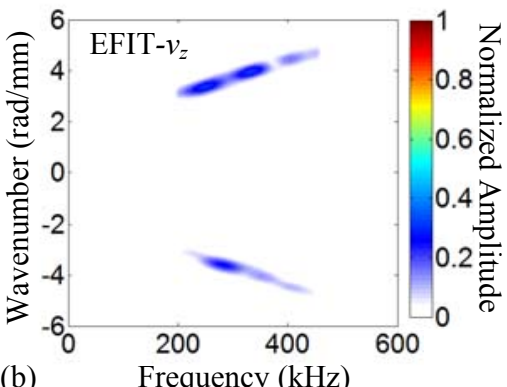

(b)

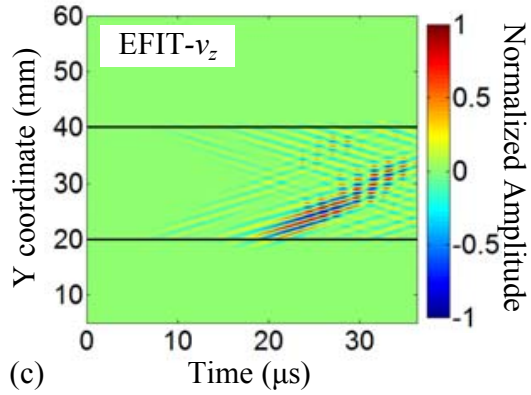

(c) spectrum; (c) the filtered wavefield.

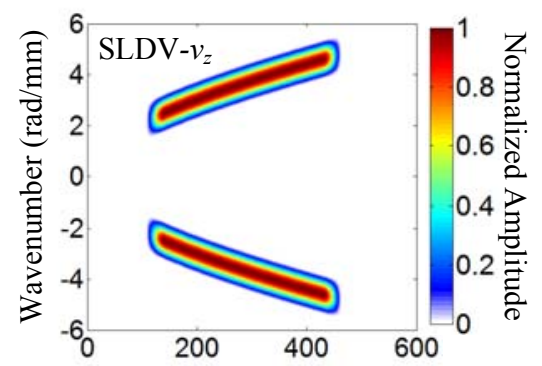

(a)

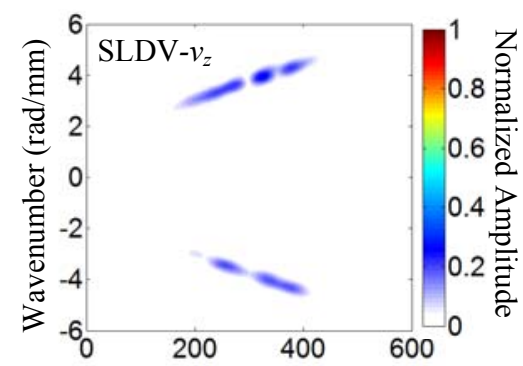

(b)

Frequency $(\mathrm{kHz})$

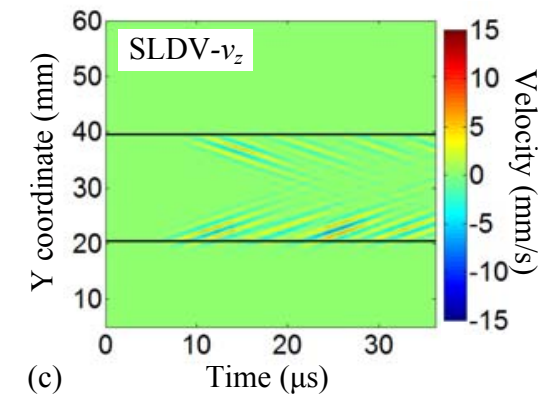

(c)

Figure 11. Delamination detection by using SLDV measurements: (a) the $f-k$ filter; (b) the filtered $f-k$ spectrum; (c) the filtered wavefield.

\section{CONCLUSIONS}

This paper presents our studies of guided wave interactions with delamination damage, as well as delamination quantification techniques for laminated composite plates. The 3D EFIT simulations were implemented to acquire both in-plane and out-of-plane wave motions for guided wave interactions with delamination damage. Both the in-plane and out-of-plane wavefields show waves bouncing back and forth between the delamination boundaries, and distinctively exhibit the "trapped waves" phenomenon. The observed behavior agrees with the wavefield observations presented by Sohn et al [27]. The wavefields were further analyzed by using $f-k$ analysis to unveil the implicit wave propagation characteristics such as $f-k$ components and wave mode contents. In the $f-k$ spectra, new $f-k$ components were observed when the guided waves interact with the delamination damage. It is also found that these new $f-k$ components are correlated to the waves propagating in the layers above the delamination.

In addition to the EFIT simulations, the SLDV experimental tests were performed. The SLDV wavefields verify the delamination induced wave interaction and "trapped waves" observed in the EFIT out-of-plate wavefields. Additionally, the SLDV $f-k$ spectra verify delamination induced new $f-k$ components observed in the EFIT out-of-plane $f-k$ spectra.

A delamination detection method using $f-k$ filtering was studied. By filtering the new $f-k$ components, the waves in the delamination region can be extracted. The filtered wavefield provides an easy means to detect and quantify the size of delamination damage. The delamination detection method was successfully applied to both the EFIT and SLDV data. Though this study was for an idealized simple delamination, it is expected that the $f-k$ analysis can be extended to characterize delamination damage in more complex, and realistic scenarios.

\section{ACKNOWLEDGEMENT}

The authors would like to thank (1) the non-reimbursement space act umbrella agreement SAA1-1181 between South Carolina Research Foundation (SCRF) and the National Aeronautics and Space Administration (NASA) Langley 
Research Center, (2) University of South Carolina SPARC graduate fellowship, and (3) SC NASA EPSCoR Research and Education Awards Program (REAP) 21-NE-USC_Yu-RGP.

\section{REFERENCES}

[1] Ip, K. H., and Mai, Y. W., "Delamination Detection in Smart Composite Beams Using Lamb Waves," Smart Materials \& Structures, 13(3), 544-551 (2004).

[2] Staszewski, W. J., Mahzan, S., and Traynor, R., "Health Monitoring of Aerospace Composite Structures-Active and Passive Approach," composites Science and Technology, 69, 1678-1685 (2009).

[3] Worlton, D. C., "Ultrasonic Testing with Lamb Waves," Nondestructive Testing, 15, 218-222 (1957).

[4] Alleyne, D. N., and Cawley, P., "The Interaction of Lamb Waves with Defects," Ieee Transactions on Ultrasonics Ferroelectrics and Frequency Control, 39, 381-397 (1992).

[5] Rose, J. L., [Ultrasonic Waves in Solid Media] Cambridge University Press, Cambridge(1999).

[6] Boller, C., "Next Generation Structural Health Monitoring and Its Integration into Aircraft Design," International Journal of Systems Science, 31, 1333-1349 (2000).

[7] Staszewski, W. J., Boller, C., and Tomlinson, G. R., [Health Monitoring of Aerospace Structures] John Wiley \& Sons, Chichester(2004).

[8] Giurgiutiu, V., [Structural Health Monitoring with Piezoelectric Wafer Active Sensors] Academic Press, Boston, MA(2008).

[9] Ong, W. H., and Chiu, W. K., "Redirection of Lamb Waves for Structural Health Monitoring," Smart Materials Research, 2012, 718686 (2012).

[10] Prasad, S. M., Balasubramaniam, K., and Krishnamurthy, C. V., "Structural Health Monitoring of Composite Structures Using Lamb Wave Tomography," Smart Materials \& Structures, 13(5), N73-N79 (2004).

[11] Wang, L., and Yuan, F. G., "Group Velocity and Characteristic Wave Curves of Lamb Waves in Composites: Modeling and Experiments," Composites Science and Technology, 67(7-8), 1370-1384 (2007).

[12] Kundu, T., Das, S., Martin, S. A., and Jata, K. V., "Locating Point of Impact in Anisotropic Fiber Reinforced Composite Plates," Ultrasonics, 48(3), 193-201 (2008).

[13] Su, Z. Q., Cheng, L., Wang, X. M., Yu, L., and Zhou, C., "Predicting Delamination of Composite Laminates Using an Imaging Approach," Smart Materials \& Structures, 18, 074002 (2009).

[14] Castaings, M., and Hosten, B., "Ultrasonic Guided Waves for Health Monitoring of High-Pressure Composite Tanks," Ndt \& E International, 41(8), 648-655 (2008).

[15] Salamone, S., Bartoli, I., di Scalea, F. L., and Coccia, S., "Guided-Wave Health Monitoring of Aircraft Composite Panels under Changing Temperature," Journal of Intelligent Material Systems and Structures, 20(9), 1079-1090 (2009).

[16] Salas, K. I., and Cesnik, C. E. S., "Guided Wave Structural Health Monitoring Using Clover Transducers in Composite Materials," Smart Materials \& Structures, 19, 015014 (2010).

[17] Song, F., Huang, G. L., and Hu, G. K., "Online Guided Wave-Based Debonding Detection in Honeycomb Sandwich Structures," Aiaa Journal, 50(2), 284-293 (2012).

[18] Giurgiutiu, V., and Soutis, C., "Enhanced Composites Integrity through Structural Health Monitoring," Applied Composite Materials, 19, 813-829 (2012).

[19] Leckey, C. A. C., Rogge, M. D., and Parker, R., "Guided Waves in Anisotropic and Quasi-Isotropic Aerospace Composites: Three-Dimensional Simulation and Experiment," Ultrasonics, 54, 385-394 (2014).

[20] Guo, N., and Cawley, P., "The Interaction of Lamb Waves with Delaminations in Composite Laminates," Journal of Acoustic Society of America, 94, 2240-2246 (1993).

[21] Petculescu, G., Krishnaswamy, S., and Achenbach, J. D., "Group Delay Measurements Using Modally Selective Lamb Wave Transducers for Detection and Sizing of Delaminations in Composites," Smart Materials \& Structures, 17, 015007 (2008).

[22] Purekar, A. S., and Pines, D. J., "Damage Detection in Thin Composite Laminates Using Piezoelectric Phased Sensor Arrays and Guided Lamb Wave Interrogation," Journal of Intelligent Material Systems and Structures, 21(10), 995-1010 (2010).

[23] Michaels, T. E., Michaels, J. E., and Ruzzene, M., "Frequency-Wavenumber Domain Analysis of Guided Wavefields," Ultrasonics, 51, 452-466 (2011). 
[24] Yeum, C. M., Sohn, H., Ihn, J. B., and Lim, H. J., "Instantaneous Delamination Detection in a Composite Plate Using a Dual Piezoelectric Transducer Network," Composite Structures, 94, 3490-3499 (2012).

[25] Hayashi, T., and Kawashima, K., "Multiple Reflections of Lamb Waves at a Delamination," Ultrasonics, 40, 193197 (2002).

[26] Ramadas, C., Balasubramaniam, K., Joshi, M., and Krishnamurthy, C. V., "Interaction of the Primary AntiSymmetric Lamb Mode (A0) with Symmetric Delaminations: Numerical and Experimental Studies," Smart Materials \& Structures, 18, 085011 (2009).

[27] Sohn, H., Dutta, D., Yang, J. Y., Park, H. J., DeSimio, M. P., Olson, S. E., and Swenson, E. D., "Delamination Detection in Composites through Guided Wave Field Image Processing," Composites Science and Technology, 71, 1250-1256 (2011).

[28] Sohn, H., Dutta, D., Yang, J. Y., DeSimio, M., Olson, S., and Swenson, E., “Automated Detection of Delamination and Disbond from Wavefield Images Obtained Using a Scanning Laser Vibrometer," Smart Materials \& Structures, 20, 045017 (2011).

[29] Glushkov, E., Glushakova, N., Golub, M. V., Moll, J., and Fritzen, C. P., "Wave Energy Trapping and Localization in a Plate with a Delamination," Smart Materials \& Structures, 21, 125001 (2012).

[30] Yu, L., and Leckey, C., "Lamb Wave-Based Quantitative Crack Detection Using a Focusing Array Algorithm," Journal of Intelligent Material Systems and Structures, 24(9), 1138-1152 (2013).

[31] Yu, L., Leckey, C., and Tian, Z., "Study on Crack Scattering in Aluminum Plates with Lamb Wave FrequencyWavenumber Analysis," Smart Materials \& Structures, 22, 065019 (2013).

[32] Halkjaer, S., [Elastic Wave Propagation in Anisotropic Inhomogeneous Materials] Technical University of Denmark, Denmark(2000).

[33] Pavlakovic, B., Lowe, M., Alleyne, D. N., and Cawley, P., "Disperse: A General Purpose Program for Creating Dispersion Curves," Review of Progress in Quantitative Nondestructive Evaluation, 16, 185-192 (1997).

[34] Rogge, M. D., and leckey, C. A. C., "Characterization of Impact Damage in Composite Laminates Using Guided Wavefield Imaging and Local Wavenumber Domain Analysis," Ultrasonics, 53(7), 1217-1226 (2013).

[35] Alleyne, D. N., and Cawley, P., "A Two Dimensional Fourier Transform Method for the Measurement of Propagating Multimode Signals," Journal of Acoustic Society of America, 89(3), 1159-1168 (1991).

[36] Ruzzene, M., "Frequency-Wavenumber Domain Filtering for Improved Damage Visualization," Smart Materials and Structures, 16, 2116-2129 (2007).

[37] Yu, L., and Tian, Z., "Lamb Wave Structural Health Monitoring Using a Hybrid Pzt-Laser Vibrometer Approach," Structural Health Monitoring, 12, 469-483 (2013).

[38] Johnson, D. H., and Dudgeon, D. E., [Array Signal Processing: Concepts and Techniques] Prentice-Hall Inc., Upper Saddle River, New Jersey, USA(1993).

[39] Tian, Z., and Yu, L., "Lamb Wave Frequency-Wavenumber Analysis and Decomposition," Journal of Intelligent Material Systems and Structures, in press (2014). 